\title{
Intima-media thickness of the common carotid arteries as a marker of retinopathy and nephropathy in sickle cell disease
}

\author{
Oluwagbemiga O. Ayoola ${ }^{1,2}$, Rahman A. Bolarinwa ${ }^{3}$, Oluwatoyin H. Onakpoya ${ }^{4}$, \\ Stephen 0. Onigbinde ${ }^{2}$, Christianah M. Asaleye ${ }^{1,2}$, Akinola A. Odedeyi ${ }^{2}$ \\ ${ }^{1}$ Department of Radiology, Faculty of Clinical Sciences, Obafemi Awolowo University, Ile- \\ Ife; ${ }^{2}$ Department of Radiology, Obafemi Awolowo University Teaching Hospital, Ile-Ife; \\ ${ }^{3}$ Department of Hematology and Blood Transfusion, Faculty of Basic Sciences, Obafemi \\ Awolowo University, Ile-Ife; ${ }^{4}$ Department of Ophthalmology, Faculty of Clinical Sciences, \\ Obafemi Awolowo University, Ile-Ife, Nigeria
}

Purpose: This study was conducted to test the hypothesis that the carotid intima-media thickness (CIMT) is higher in patients with sickle cell disease (SCD) than in the normal population, and to determine the relationships of the CIMT with central retinal artery (CRA) and renal artery Doppler indices.

Methods: Forty-four confirmed steady-state SCD patients aged 16 years and above were recruited consecutively. The Doppler velocimetric indices of their right renal artery and both CRAs were obtained. The CIMT was also measured on each side via B-mode ultrasonography. The subjects were categorized by age and sex. Mean and median values for each group were determined. The Spearman correlation test was used to quantify the relationships between CIMT and the Doppler parameters.

Results: The participants had a median age of 24.50 years (interquartile range, 12.50 to 36.50 years). Twenty-three were men (52.3\%) and 21 were women (47.7\%). The median CIMT was $0.70 \mathrm{~mm}$ (IQR, 0.50 to $0.90 \mathrm{~mm}$ ). Significant correlations with the CIMT were found for the CRA peak systolic velocity $(r=0.312, P=0.003)$, the renal artery resistivity index ( $R I)(r=0.284$, $P=0.007)$, and the renal artery pulsatility index $(P I)(r=0.273, P=0.010)$. There was no significant relationship between the CIMT and the CRA end-diastolic velocity, CRA RI, or CRA PI.

Conclusion: CIMT in SCD patients was higher than in the previously reported age groups of the reference populations. In addition, the CIMT was significantly correlated with the CRA peak systolic velocity, the renal artery $\mathrm{RI}$, and the renal artery PI.

Keywords: Central retinal artery; Carotid intima-media thickness, Renal artery Doppler indices; vaso-occlusion

\section{ORIGINAL ARTICLE}

https://doi.org/10.14366/usg.19016 pISSN: 2288-5919 • elSSN: 2288-5943 Ultrasonography 2020;39:79-84

Received: February 15, 2019

Revised: June 7, 2019

Accepted: July 5, 2019

Correspondence to:

Stephen O. Onigbinde, MBCHB, Department of Radiology, Obafemi Awolowo University Teaching Hospital, Ile-Ife, Osun State, P.M.B 5538, Nigeria

Tel, Fax. +234-8163988958

E-mail:drstevola@gmail.com

This is an Open Access article distributed under the terms of the Creative Commons Attribution NonCommercial License (http://creativecommons.org licenses/by-nc/4.0/) which permits unrestricted noncommercial use, distribution, and reproduction in any medium, provided the original work is properly cited.

Copyright (C) 2020 Korean Society of Ultrasound in Medicine (KSUM)

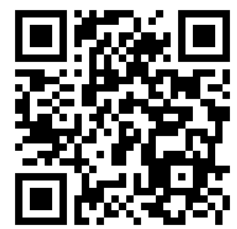

How to cite this article:

Ayoola 00, Bolarinwa RA, Onakpoya $\mathrm{OH}$ Onigbinde SO, Asaleye CM, Odedeyi AA Intima-media thickness of the common carotid arteries as a marker of retinopathy and nephropathy in sickle cell disease. Ultrasonography. 2020 Jan;39(1):79-84. 


\section{Introduction}

Vascular occlusion, caused by sickled red and white blood cells that are abnormally adherent to the vascular endothelium [1], is a major cause of morbidity in sickle cell disease (SCD) via a variety of generally nonfatal complications such as painful crisis, aseptic necrosis of bone, priapism, leg ulcers, and proliferative retinopathy [2].

Central retinal artery (CRA) occlusion, which is the ocular analog of cerebral stroke, is an ophthalmological emergency that can result in profound monocular vision loss. The risk factors for CRA occlusion are the same atherosclerosis-related factors that contribute to the risk of stroke and heart disease [3]. The incidence of CRA occlusion is estimated to be 1 per 100,000 per year and it accounts for 1 in 10,000 ophthalmological outpatient visits [4]. Retinal vascular occlusions typically occur in the peripheral retina, leading to vascular proliferation and fibrosis characteristic of sickle cell retinopathy [5]. Ipsilateral carotid artery atherosclerosis is the most common cause of retinal artery occlusion, with a prevalence as high as 70\% reported among patients with CRA occlusion. Other causes include hematological conditions such as SCD, lymphoma, and leukemia [6].

B-mode ultrasonography is a safe, non-invasive diagnostic tool that can be used to measure the carotid intima-media thickness (CIMT), thereby providing information that can be used in risk stratification for cardiovascular and stroke events [7].

The early and quantitative assessment of small-vessel disease, such as pathological processes in the CRA (which measures approximately 0.15 to $0.2 \mathrm{~mm}$ in diameter distally) is important. Doppler flowmetry of the CRA seems appropriate for quantitative assessment, as it causes minimal discomfort, requires little time, and has high reproducibility [8].

Retinal vaso-occlusive disorder can occur in patients with chronic renal failure in the absence of other chronic diseases that predispose to it [9]. End-stage renal disease, which can be caused by SCD, is associated with uremia-related risk factors (hemodynamic overload, anemia, hyperhomocysteinemia, and increased oxidative stress) for atherogenesis [10], which underlies atherosclerosis.

We hypothesized that the CIMT would be higher in patients with SCD than in the normal population, and that the CIMT would be positively correlated with CRA Doppler flowmetry indices in patients with SCD.

\section{Materials and Methods}

In this prospective cohort study, we recruited consecutive 44 patients with steady-state SCD aged 16 years and above from the hematology outpatient clinic of our institution. HbSS genotype confirmation was done by hemoglobin gel electrophoresis. The study was approved by the research and ethics committee of the hospital and the study was carried out in compliance with the Declaration of Helsinki of 1964 and its subsequent revisions from March 2013 through December 2013. A written informed consent was obtained from all study subjects. The study subjects had been previously studied with respect to the possible use of femoral artery intima-media thickness as a biomarker of chronic leg ulcer [11]. In contrast, in the present study, the CIMT was studied as a marker of retinopathy and nephropathy in SCD patients.

Excluded from this study were patients with congenital urogenital anomalies; patients with urinary tract infection, diabetes mellitus, systemic hypertension, dyslipidemia, a history of ocular disease or a previous ophthalmological operation; patients on oral contraceptive pills or adrenergic drugs; smokers; human immunodeficiency viruspositive individuals; patients on hemodialysis or with massive edema; patients on any medications potentially interfering with renal function, such as cimetidine, probenecid, and angiotensinconverting enzyme inhibitors; patients with space-occupying lesions of the kidney, urinary or renal vascular obstructions, urinary tract infection, or a history of renal surgery; and those who had a single kidney. None of the SCD subjects were on hydroxyurea or Nicosan/ Hemoxin.

\section{Ultrasonography Technique}

Ultrasonographic examinations were performed using a Mindray real-time ultrasound machine (model DC-7, Shenzhen Mindray Bio-medical Electronics, Nanshan, Shenzhen, China) using a linear transducer (frequency, 7.5-12.0 MHz) for ocular and carotid scans. A curvilinear transducer (frequency, 3.5-5 MHz) was used for the renal scans.

The CRA Doppler parameters were obtained according to the protocol described by Baxter and Williamson [12] (Fig. 1), while the ultrasonographic examination of the CIMT was carried out using the protocol described by Pignoli et al. [13]. Measurements were taken 3 times, unfreezing the image at each occasion and relocating the position of maximal intima-media thickness (Fig. 2).

The right renal artery of all subjects was examined with the subjects in the supine position. A B-mode ultrasonographic examination was first performed on each subject to evaluate them for exclusion criteria related to the genitourinary system. Colorcoded duplex ultrasound interrogation was used to identify the intraparenchymal arteries. Resistivity index (RI) and pulsatility index (PI) values were obtained from the right interlobar or arcuate arteries. Only parameters from the right kidney were obtained in this study, as it has been shown that there is no significant difference between the RI and PI values of the right and left kidneys [14]. The 


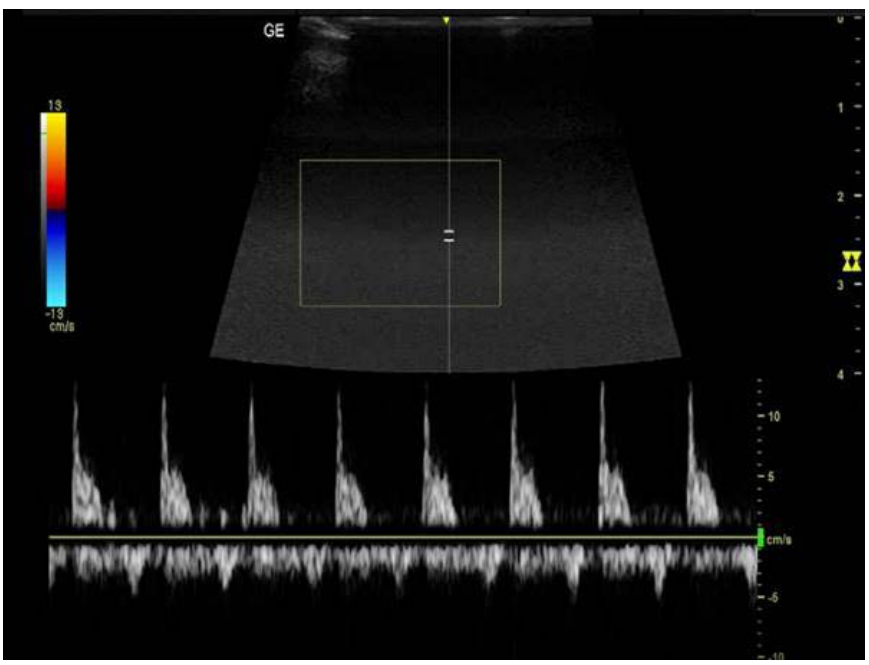

Fig. 1. Triplex ultrasound image of the central retinal artery showing a narrowed and spiked peak systolic waveform, a relatively high peak systolic velocity in comparison to the enddiastolic velocity, and a resistivity index of 0.89 .

mean values of the Doppler indices were calculated from at least three consecutive waveforms obtained from the three different regions of the kidney while subjects held their breath at the end of respiration. The interlobar or arcuate arteries were chosen for this study because their waveforms show less variability. The Doppler sample volume was set at a 2- to 4-mm gate just appropriate to be placed in the mid-portion of the diameter of the vessel to be insonated. The waveforms were optimized with a minimum Doppler angle of $<60^{\circ}$ adapted for maximum spectral amplitudes. The peak systolic velocity (PSV) and end-diastolic velocity (EDV) were measured and the RI and PI were automatically calculated using the existing software (automated algorithm) capabilities of the scanner from the following formulae:

Resitive index (RI)=Peak systolic frequency shift-minimum diastolic frequency shift:Peak systolic frequency shift during the whole cardiac cycle

Pulsatility Index (PI)=Peak systolic frequency shift-minimum diastolic frequency shift:Mean frequency shift during the whole cardiac cycle

\section{Statistical Analysis}

The data were analyzed using SPSS version 22 for Windows (IBM Corp., Armonk, NY, USA). The normality of the data was determined using the Kolmogorov-Smirnov test. The measure of central tendency for each continuous variable was presented as either the mean or median with interquartile range (IQR). The relationships of the CIMT

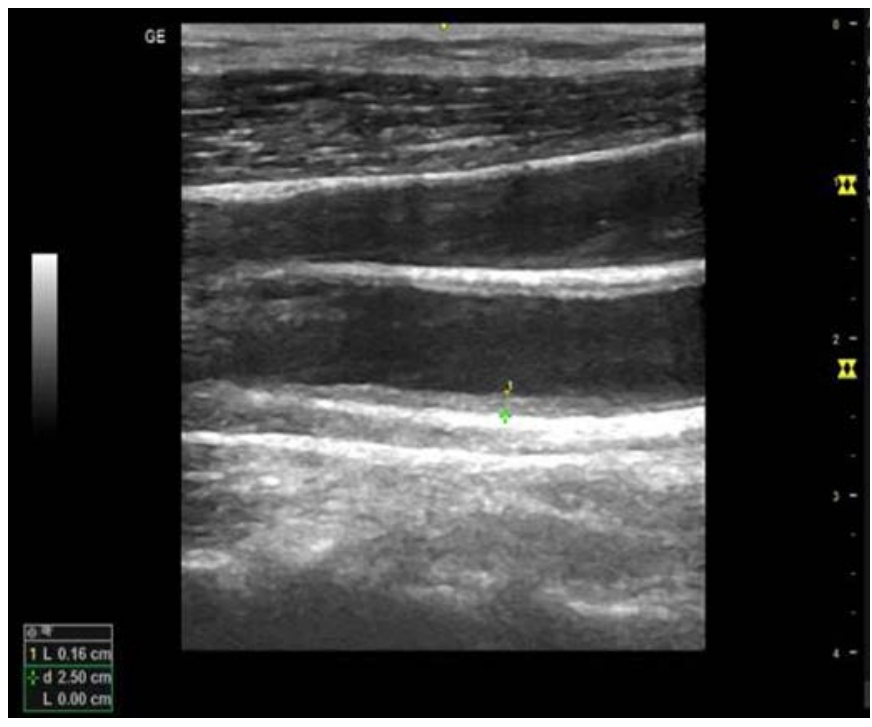

Fig. 2. B-mode ultrasound image of the common carotid artery, showing thickened intima media.

with the CRA and renal artery Doppler velocimetric indices were assessed using Spearman correlation analysis, linear regression, and logistic regression as appropriate. The level of statistical significance was set at $\mathrm{P}<0.05$.

\section{Results}

Eighty-eight CIMT measurements from 44 patients with SCD were evaluated. The patients had a median age of 24.50 years (IQR, 12.50 to 36.50 years). The group was composed of 23 men (52.3\%) and 21 women (47.7\%). The median CIMT was $0.70 \mathrm{~mm}$ (IQR, 0.50 to $0.90 \mathrm{~mm}$ ).

The median CIMTs of SCD patients in the present study looked higher than in the previously reported reference populations [15], categorized by age group and sex (Table 1). The median values of ocular and renal Doppler velocimetric parameters are shown in Table 2. There was no significant difference between the median right and left CIMT. The median CIMT on the left side was $0.7 \mathrm{~mm}$ (IQR, 0.40 to $1.00 \mathrm{~mm}$ ), while that on the right was $0.75 \mathrm{~mm}(\mathrm{IQR}, 0.55$ to 0.95 $\mathrm{mm} ; \mathrm{P}=0.128$ ). In addition, no significant difference was found in the median CIMT between male and female study participants (male median CIMT, $0.70 \mathrm{~mm}$ [IQR, 0.50 to $0.90 \mathrm{~mm}$ ]; female median CIMT, $0.75 \mathrm{~mm}$ [IQR, 0.35 to $1.15 \mathrm{~mm}$ ]; $\mathrm{P}=0.969)$. The median CRA PSV of male participants, $9.57 \mathrm{~cm} / \mathrm{sec}$ (IQR, 4.44 to $14.70 \mathrm{~cm} /$ $\mathrm{sec})$, was higher than that of female participants $(8.43 \mathrm{~cm} / \mathrm{sec} ; \mathrm{IQR}$, 5.35 to $11.51 \mathrm{~cm} / \mathrm{sec})$; however, this difference was not statistically significant $(P=0.427)$.

Table 3 shows the correlations of the CIMT with CRA PSV, CRA 
Table 1. Median CIMT and mean CIMT compared to mean reference values according to age group and sex

\begin{tabular}{ccccc}
\hline \multirow{2}{*}{ Age group $(\mathrm{yr})$} & No. & Median of subjects $(\mathrm{mm})$ & \multicolumn{2}{c}{ Mean $(\mathrm{mm})$} \\
\cline { 4 - 5 } & & & & Subject \\
\hline Male (n=23, 46 CIMT measurements) & 26 & 0.700 & 0.762 & 0.676 \\
$0-24$ & 18 & 0.750 & 0.828 & 0.730 \\
$25-34$ & 2 & 0.800 & 0.800 & 0.749 \\
$35-44$ & 0 & - & - & 0.824 \\
$45-54$ & 18 & & & 0.614 \\
Female (n=21, 42 CIMT measurements) & 0.650 & 0.756 & 0.690 \\
$0-24$ & 12 & 0.750 & 0.775 & 0.665 \\
$25-34$ & 8 & 0.750 & 0.800 & 0.775 \\
$35-44$ & 4 & 1.000 & 0.975 & \\
$45-54$ & & & \\
\hline
\end{tabular}

CIMT, carotid intima-media thickness.

Table 2. Median CRA and RA Doppler parameters

\begin{tabular}{lcl}
\hline \multicolumn{1}{c}{ Variable } & No. & \multicolumn{1}{c}{ Median (IQR) } \\
\hline CRA PSV $(\mathrm{cm} / \mathrm{sec})$ & 88 & $5.19(3.28-13.66)$ \\
CRA EDV $(\mathrm{cm} / \mathrm{sec})$ & 88 & $2.14(1.00-3.28)$ \\
CRA PI & 88 & $1.53(0.88-2.18)$ \\
CRA RI & 88 & $0.75(0.62-0.88)$ \\
RA PI & 44 & $1.01(0.69-1.33)$ \\
RA RI & 44 & $0.64(0.51-0.77)$ \\
\hline
\end{tabular}

CRA, central retinal artery; RA, renal artery; IQR, interquartile range; $P S V_{\text {, peak }}$ systolic velocity; EDV, end-diastolic velocity; PI, pulsatility index; RI, resistivity index.

EDV, CRA PI, CRA RI, renal artery PI, and renal artery RI. Of the ocular and renal Doppler parameters, the CRA PSV, renal artery RI, and renal artery PI had weak, but significant, positive correlations with CIMT.

CRA PSV was correlated with renal artery RI, but not with renal artery PI (renal artery PI: $r=0.05, P=0.212$; renal artery RI: $r=0.271$, $\mathrm{P}=0.012)$.

\section{Discussion}

The CIMT is the combined thickness of the intimal and medial layers of the carotid artery [16]. In addition to atherosclerosis, the CIMT can also be increased by other factors such as age and hypertension, which were accounted for in this study [16]. The CIMT can be used to predict atherosclerosis and cardiac events. An increased CIMT, irrespective of atherosclerosis, is associated with an elevated risk of stroke and myocardial infarction [17]. The CIMT has been found to be an acceptable marker for subclinical atherosclerosis [16].

SCD causes a chronic anemic state, which is compensated for
Table 3. Correlations of the CIMT with ocular and renal Doppler parameters

\begin{tabular}{llcc}
\hline & No. & \multicolumn{2}{c}{ CIMT } \\
\cline { 3 - 4 } & & $\mathrm{R}$ & P-value \\
\hline CRA PSV & 88 & 0.312 & 0.003 \\
CRA EDV & 88 & 0.070 & 0.523 \\
CRA PI & 88 & 0.149 & 0.170 \\
CRA RI & 88 & 0.195 & 0.072 \\
RA PI & 88 & 0.273 & 0.010 \\
RA RI & 88 & 0.284 & 0.007 \\
\hline
\end{tabular}

CIMT, carotid intima-media thickness; $R$, Spearman correlation coefficient; CRA, central retinal artery; PSV, peak systolic velocity; EDV, end-diastolic velocity; PI, pulsatility index; RI, resistivity index; RA, renal artery.

by increased cardiac output accompanied by increased blood mass and blood pressure. There is subsequent stiffening, enlargement, and remodeling of the central elastic arteries such as the common carotid artery [18]. Sickle cell anemia affects both the microvascular circulation and large vessels, such as the carotid arteries [19]. The complications of sickle cell anemia, such as stroke, can be explained by occlusive vasculopathy, which in turn is due to intima-media thickening and thrombus formation [20]. Intima-media thickness has been identified as the most important factor associated with stroke in SCD patients [2]. It may be associated with abnormal adhesive and procoagulant effects of the red blood cells of SCD patients. Other mechanisms of vasculopathy include microvascular occlusion by sickle cell erythrocytes, bone marrow/fat embolism, vasospasm, and/or vasodilatation. Intima-media changes may also be explained by high flow turbulence at areas of arterial bifurcation and tortuosity. In addition, such changes may occur due to increased blood flow secondary to anemia. Intima-media proliferation has 
been reported in arteries of the spleen, lungs, penis, and kidneys; however, the frequency and significance of those changes have not been well documented. These widespread changes may contribute to other vascular complications. Intimal hyperplasia causes changes by direct occlusion and superimposed thrombosis, potentially contributing to the trapping of sickled red blood cells in the microcirculation by slowing microvascular flow and increasing the capillary transit time [2]. In this study, the mean CIMT of all the subjects studied was higher than that of the corresponding age and sex reference groups.

Sickle cell retinopathy is an ocular manifestation of SCD [21]. Retinal changes can be considered as a focal neurologic change in the definition of stroke [22]. The ocular manifestations of extracranial cerebrovascular pathology or chronic ischemic diseases commonly occur with a stroke-like temporal profile. These manifestations can be subdivided into hemorrhagic retinopathy, also called venous stasis retinopathy, and ischemic oculopathy [23]. Of all the Doppler velocimetric parameters of the CRA studied, only the CRA PSV had a significant correlation with the CIMT.

SCD involves structural, tubular, and hemodynamic changes that are associated with increased hormone synthesis (erythropoietin, renin, and angiotensin). These changes start in childhood and lead to chronic anemia, increased blood flow, and vaso-occlusion [24]. The renal medulla contains renal tubules and medullary blood vessels. The mechanism of hemoglobin $S$ polymerization and cell sickling accounts for much of the pathophysiology of sickle cell nephropathy [24]. Slow blood flow in the renal microvasculature provides a prolonged exposure time for the polymerization of hemoglobin $S$, which in turn leads to microvascular obstruction and subclinical renal tissue infarction, especially in the medulla [25]. Proteinuria, hypertension, and anemia in SCD patients predict end-stage renal disease, which is preceded by alterations in renal hemodynamics, such as altered effective renal plasma flow and an elevated glomerular filtration rate. The effective renal plasma flow and glomerular filtration rate are elevated in young sickle cell patients, decrease to normal levels in adolescence, and decrease further to subnormal levels as patients grow older [24]. Significant deterioration in renal function may occur long before it is detected clinically [24]. The sickling of red blood cells results in almost complete occlusion of the vasa recta and dilatation of the remaining medullary blood vessels that end blindly [24]. These renal vascular changes and subsequent tissue ischemia may partly account for multiorgan failure syndrome, defined as affecting at least two major organ systems, including the lung, liver, and/or kidney [24]. Based on the assertion of previous researchers that chronic renal failure can be caused by SCD [10] and can independently lead to vascular retinopathy [9], we evaluated the relationship between the CIMT and renal artery $\mathrm{RI}$ and $\mathrm{Pl}$, along with renal Doppler indices and retinal velocimetry. The CIMT was correlated positively with the renal artery indices. In addition, a positive correlation was found between CRA PSV and renal artery RI.

An evaluation of biochemical parameters and ophthalmological examinations would have added value to this study. The findings that SCD patients had a higher CIMT than the normal population and that in those patients, the CIMT was correlated positively with CRA PSV and renal artery RI, suggest that the CIMT is increased in SCD and can function as a marker of ocular and renal changes in SCD patients. Further elucidation of the relationship between Doppler changes and end-organ damage will lead to more effective prevention and treatment. Early detection of retinopathy and appropriate therapy in SCD patients may reduce their risk of complications.

ORCID: Oluwagbemiga 0. Ayoola: https://orcid.org/0000-0002-7832-3390; Rahman A. Bolarinwa: https://orcid.org/0000-0003-2782-7190; Oluwatoyin H. Onakpoya: https://orcid.org/0000-0001-5796-6242; Stephen 0. Onigbinde: https://orcid.org/00000002-6179-3505; Christianah M. Asaleye: https://orcid.org/0000-0002-6355-3337; Akinola A. Odedeyi: https://orcid.org/0000-0002-0003-2235

\section{Author Contributions}

Conceptualization: Ayoola 00, Onigbinde SO. Data acquisition: Ayoola 00, Bolarinwa RA. Data analysis or interpretation: Ayoola 00, Onigbinde SO. Drafting of the manuscript: Ayoola 00, Onigbinde SO. Critical revision of the manuscript: Ayoola O0, Bolarinwa RA, Onakpoya OH, Onigbinde SO, Asaleye CM, Odedeyi AA. Approval of the final version of the manuscript: all authors.

\section{Conflict of Interest}

No potential conflict of interest relevant to this article was reported.

\section{Acknowledgments}

This research was partially funded by the Obafemi Awolowo University Teaching Hospital, Ile-Ife, Osun State, Nigeria. We wish to acknowledge Obafemi Awolowo University Hospital for partly funding this research.

\section{References}

1. Mohandas N, Evans E. Sickle erythrocyte adherence to vascular endothelium. Morphologic correlates and the requirement for divalent cations and collagen-binding plasma proteins. J Clin Invest 1985:76:1605-1612.

2. Francis RB Jr, Johnson CS. Vascular occlusion in sickle cell disease: current concepts and unanswered questions. Blood 1991;77:14051414. 
3. Cugati S, Varma DD, Chen CS, Lee AW. Treatment options for central retinal artery occlusion. Curr Treat Options Neurol 2013;15:63-77.

4. Rumelt S, Dorenboim Y, Rehany U. Aggressive systematic treatment for central retinal artery occlusion. Am J Ophthalmol 1999;128:733738.

5. Fine LC, Petrovic V, Irvine AR, Bhisitkul RB. Spontaneous central retinal artery occlusion in hemoglobin sickle cell disease. Am J Ophthalmol 2000;129:680-681.

6. Ahuja RM, Chaturvedi S, Eliott D, Joshi N, Puklin JE, Abrams GW. Mechanisms of retinal arterial occlusive disease in African American and Caucasian patients. Stroke 1999;30:1506-1509.

7. Darabian S, Hormuz M, Latif MA, Pahlevan S, Budoff MJ. The role of carotid intimal thickness testing and risk prediction in the development of coronary atherosclerosis. Curr Atheroscler Rep 2013;15:306.

8. Hiroki M, Miyashita K, Yoshida H, Hirai S, Fukuyama H. Central retinal artery Doppler flow parameters reflect the severity of cerebral small-vessel disease. Stroke 2003;34:e92-e94.

9. Stoumbos VD, Klein ML, Goodman S. Purtscher's-like retinopathy in chronic renal failure. Ophthalmology 1992;99:1833-1839.

10. Arici $\mathrm{M}$, Walls J. End-stage renal disease, atherosclerosis, and cardiovascular mortality: is C-reactive protein the missing link? Kidney Int 2001;59:407-414.

11. Ayoola O0, Bolarinwa RA, Onakpoya UU, Adedeji TA, Onwuka CC, Idowu BM. Intima-media thickness of the common femoral artery as a marker of leg ulceration in sickle cell disease patients. Blood Adv 2018;2:3112-3117.

12. Baxter GM, Williamson TH. Color Doppler imaging of the eye: normal ranges, reproducibility, and observer variation. J Ultrasound Med 1995;14:91-96.

13. Pignoli P, Tremoli E, Poli A, Oreste P, Paoletti R. Intimal plus medial thickness of the arterial wall: a direct measurement with ultrasound imaging. Circulation 1986;74:1399-1406.
14. Terry JD, Rysavy JA, Frick MP. Intrarenal Doppler: characteristics of aging kidneys. J Ultrasound Med 1992;11:647-651.

15. Jarauta E, Mateo-Gallego R, Bea A, Burillo E, Calmarza P, Civeira F. Carotid intima-media thickness in subjects with no cardiovascular risk factors. Rev Esp Cardiol 2010;63:97-102.

16. Cobble M, Bale B. Carotid intima-media thickness: knowledge and application to everyday practice. Postgrad Med 2010;122:10-18.

17. Canga A, Kocaman SA, Durakoglugil ME, Cetin M, Erdogan T, Cicek $Y$, et al. Increased carotid and brachial intima-media thickness is related to diffuse coronary involvement rather than focal lesions. Angiology 2013;64:356-363.

18. Metivier F, Marchais SJ, Guerin AP, Pannier B, London GM. Pathophysiology of anaemia: focus on the heart and blood vessels. Nephrol Dial Transplant 2000;15 Suppl 3:14-18.

19. de Montalembert M, Aggoun Y, Niakate A, Szezepanski I, Bonnet D. Endothelial-dependent vasodilation is impaired in children with sickle cell disease. Haematologica 2007;92:1709-1710.

20. Adams R, McKie V, Nichols F, Carl E, Zhang DL, McKie K, et al. The use of transcranial ultrasonography to predict stroke in sickle cell disease. N Engl J Med 1992;326:605-610.

21. Tantawy AA, Andrawes NG, Adly AA, El Kady BA, Shalash AS. Retinal changes in children and adolescents with sickle cell disease attending a paediatric hospital in Cairo, Egypt: risk factors and relation to ophthalmic and cerebral blood flow. Trans $\mathrm{R}$ Soc Trop Med Hyg 2013;107:205-211.

22. Gorelick PB, Testai FD, Hankey GJ, Wardlaw JM. Hankey's clinical neurology. Boca Raton, FL: CRC Press, 2014.

23. Carter JE. Chronic ocular ischemia and carotid vascular disease. Stroke 1985;16:721-728.

24. Ataga Kl, Orringer EP. Renal abnormalities in sickle cell disease. Am J Hematol 2000;63:205-211.

25. John N. A review of clinical profile in sickle cell traits. Oman Med J 2010;25:3-8. 